\title{
A Lower Lipschitz Condition for the Stable Subordinator
}

\author{
JOHN HAWKES
}

\section{Introduction}

In [5: pp. 168-172] Lévy showed that if $X_{t}(\omega)$ is the Brownian motion process in $R$ and if

$$
\phi(h)=(2 h \log 1 / h)^{\frac{1}{2}}
$$

then

almost surely.

$$
\lim _{\varepsilon \rightarrow 0} \sup _{\substack{0 \leqq t \leqq 1 \\ 0<h<\varepsilon}} \frac{\left|X_{t+h}(\omega)-X_{t}(\omega)\right|}{\phi(h)}=1
$$

Since the Brownian motion process is the only stable process with continuous sample paths we can have no such result for stable processes of index $\alpha, \alpha<2$. We can, however, pose a related question for the stable subordinator, $T_{t}(\omega)$. Here the sample paths are increasing and one can ask to what extent they are uniformly increasing. In other words, for what functions $\phi$, if any, do we have

holding almost surely?

$$
\lim _{\varepsilon \rightarrow 0} \inf _{\substack{0 \leqq t \leqq 1 \\ 0<h<\varepsilon}} \frac{T_{t+h}(\omega)-T_{t}(\omega)}{\phi(h)}=1
$$

In this paper we answer this question and then use the relationship between the local time at zero of a stable process of index $\alpha, \alpha>1$, in $R$ and the stable subordinator of index $1-1 / \alpha$ to obtain a modulus of continuity result for the local time. Finally, we apply our result to a problem considered by Jain and Pruitt in [4].

\section{Preliminaries}

Suppose that $0<\alpha \leqq 2$. A stable process of index $\alpha$ in $R$ is a stochastic process $X_{t}(\omega)$, defined on some probability space $(\Omega, \mathscr{F}, P)$ with $X_{0}(\omega)=0$, stationary independent increments, and with characteristic function given by

$$
E \exp i\left(z X_{t}\right)=\exp [-t \psi(z)] \text {. }
$$

Here $\psi(z)=c|z|^{\alpha}[1-i h \operatorname{sgn}(z) w(\alpha, z)]$, where $w(\alpha, z)=\tan (\pi \alpha / 2)$ if $\alpha \neq 1$, and $w(1, z)=-(2 \log |z|) / \pi . h$ and $c$ are real constants satisfying $|h| \leqq 1$ and $c>0$. If $\alpha=2$ and $c=\frac{1}{2}, X_{t}(\omega)$ is the Brownian motion process, if $0<\alpha<1, c=\cos (\pi \alpha / 2)$ and $h=1$ the corresponding process, $T_{t}(\omega)$, has increasing sample paths and is called the stable subordinator of index $\alpha$. $T_{t}(\omega)$ is seen to satisfy

$$
E \exp \left(-\lambda T_{t}\right)=\exp \left(-t \lambda^{\alpha}\right)
$$


It is easily seen that these processes have transition densities $p(t, y-x)$ which, for each $t$, are bounded and continuous functions of $y$. When $\alpha \neq 1$ we always have for each $r>0$.

$$
r^{1 / \alpha} p\left(r t, r^{1 / \alpha} x\right)=p(t, x)
$$

Now suppose that $X_{t}(\omega)$ is a stable process of index $\alpha, \alpha>1$, in $R$. Then for any Borel set $B$ we define

$$
T(u, B, \omega)=\int_{0}^{u} I_{B}\left[X_{t}(\omega)\right] d t
$$

where $I_{B}(x)$ is the indicator function of the set $B$. Boylan [3] demonstrated the existence of a density $L(u, x, \omega)$ of $T(u, B, \omega)$ which is jointly continuous in $x$ and $u$. $L(u, x, \omega)$ is called the local time at $x$ of $X_{t}(\omega)$.

Let $A_{u}(\omega)=L(u, 0, \omega)$ be the local time at zero of $X_{t}(\omega)$. In [8] Stone showed that if $S_{t}(\omega)$ is the process defined by

$$
S_{t}(\omega)=\inf \left[u: A_{u}(\omega)>t\right]
$$

then $S_{t}(\omega)=\rho T_{t}(\omega)$, where $T_{t}(\omega)$ is the stable subordinator of index $\gamma, \gamma=1-1 / \alpha$, and $\rho$ is given by

$$
\rho^{-\gamma}=\pi^{-1} \Gamma(1+1 / \alpha) \Gamma(1-1 / \alpha) c^{-1 / \alpha} \operatorname{Re}\left\{[1-i h \tan (\pi \alpha / 2)]^{-1 / \alpha}\right\} .
$$

\section{The Distribution of $T_{t}$}

We could deduce our estimate for the distribution of $T_{t}$ from that for the density given in [7]. However, we lose nothing by doing the computation directly.

Lemma 1. Let $T_{t}(\omega)$ be a stable subordinator of index $\alpha$. Then, as $x \rightarrow 0^{+}$,

$$
P\left(T_{t} \leqq t^{1 / \alpha} x\right) \sim c_{1} x^{\alpha /(2(1-\alpha))} \exp \left(-c_{2} x^{-(\alpha /(1-\alpha))}\right) .
$$

Where

and

$$
c_{1}=c_{1}(\alpha)=\left[2 \pi(1-\alpha) \alpha^{\alpha /(2(1-\alpha))}\right]^{-\frac{1}{2}}
$$

$$
c_{2}=c_{2}(\alpha)=(1-\alpha) \alpha^{\alpha /(1-\alpha)} \text {. }
$$

Proof. Let $F(x)=P\left(T_{t} \leqq t^{1 / \alpha} x\right)=P\left(T_{1} \leqq x\right)$, and let $f(x)$ be the density of $F(x)$.

Then

$$
\begin{aligned}
\exp \left(-\lambda^{\alpha}\right) & =E \exp \left(-\lambda T_{1}\right) \\
& =\int_{0}^{\infty} \exp (-\lambda x) f(x) d x
\end{aligned}
$$

By the formula for the inversion of a Laplace transform we have

for any $a>0$.

$$
f(x)=\frac{1}{2 \pi i} \int_{a-i \infty}^{a+i \infty} \exp \left(-\lambda^{\alpha}+x \lambda\right) d \lambda
$$

Put $\lambda_{0}=(\alpha / x)^{1 /(1-\alpha)}$ and take $a=\lambda_{0}$, then

where $\phi(\mu)=-\lambda_{0}^{\alpha} \mu^{\alpha}+x \lambda_{0} \mu$.

$$
f(x)=\frac{\lambda_{0}}{2 \pi i} \int_{1-i \infty}^{1+i \infty} \exp [\phi(\mu)] d \mu
$$


Now we have

$$
\begin{aligned}
\phi(1) & =-c_{2} x^{-(\alpha /(1-\alpha))}, \\
\phi^{\prime}(1) & =0, \\
\phi^{\prime \prime}(1) & =\alpha(1-\alpha) \alpha^{\alpha /(1-\alpha)} x^{-(\alpha /(1-\alpha))} .
\end{aligned}
$$

So, if $\mu=1+i y$ and $y$ real, as $y \rightarrow 0^{+}$

$$
\phi(\mu) \doteqdot \phi(1)-\frac{1}{2} \phi^{\prime \prime}(1) y^{2} .
$$

The saddle point approximation shows that, as $x \rightarrow 0^{+}$,

$$
\begin{aligned}
f(x) & \sim \frac{\lambda_{0}}{2 \pi i} \exp [\phi(1)] i \int_{-\infty}^{\infty} \exp \left[-\frac{1}{2} \phi^{\prime \prime}(1) y^{2}\right] d y \\
& =\frac{\lambda_{0}}{2 \pi} \exp [\phi(1)] \sqrt{\frac{2 \pi}{\phi^{\prime \prime}(1)}} \\
& =A x^{-(1 /(1-\alpha))+\alpha /(2(1-\alpha))} \exp \left(-c_{2} x^{-(\alpha /(1-\alpha))}\right)
\end{aligned}
$$

where

$$
A=\frac{\alpha^{1 /(2(1-\alpha))}}{[2 \pi(1-\alpha)]^{\frac{1}{2}}} .
$$

Now $F(x)=\int_{0}^{x} f(s) d s$ so that, as $x \rightarrow 0^{+}$,

$$
F(x) \sim A \int_{0}^{x} s^{-(1 /(1-\alpha))+\alpha /(2(1-\alpha))} \exp \left(-c_{2} s^{-(\alpha /(1-\alpha))}\right) d s .
$$

Putting $u=s^{-\{\alpha /(1-\alpha))}$ we have

$$
\begin{aligned}
F(x) & \sim A \frac{1-\alpha}{\alpha} \int_{x^{-(\alpha /(1-\alpha))}}^{\infty} u^{-\frac{1}{2}} \exp \left(-c_{2} u\right) d u \\
& \sim \frac{A}{c_{2}} \frac{1-\alpha}{\alpha}\left(x^{-(\alpha /(1-\alpha))}\right)^{-\frac{1}{2}} \exp \left(-c_{2} x^{-(\alpha /(1-\alpha))}\right) \\
& =c_{1} x^{\alpha /(2(1-\alpha))} \exp \left(-c_{2} x^{-(\alpha /(1-\alpha))}\right)
\end{aligned}
$$

and the lemma is proved.

\section{Lipschitz Conditions}

We come now to our main theorem.

Theorem 1. Let $T_{t}(\omega)$ be a stable subordinator of index $\alpha$, and let

then

$$
\phi(h)=\frac{h^{1 / \alpha}}{[\log 1 / h]^{(1-\alpha) / \alpha}}
$$

almost surely.

$$
\lim _{\varepsilon \rightarrow 0} \inf _{\substack{0 \leqq i \leq 1 \\ 0<h<\varepsilon}} \frac{T_{t+h}(\omega)-T_{t}(\omega)}{\phi(h)}=c_{2}^{(1-\alpha) / \alpha}
$$


Proof. Let $q, j$ and $k$ be non negative integers such that $0 \leqq j \leqq 2^{q}$ and $[q / 3] \leqq$ $k \leqq q$, and let $\delta$ be such that $0<\delta<1$.

Let $A_{j k q}$ be the event:

$$
T_{(j+k) / 2^{q}}(\omega)-T_{j / 2^{q}}(\omega) \leqq \phi\left(\frac{k+2}{2^{q}}\right)\left[\frac{c_{2}}{1+\delta}\right]^{(1-\alpha) / \alpha}
$$

so that $P\left(A_{j k q}\right)=P\left(T_{1} \leqq x\right)$ where

$$
x=\left(\frac{2^{q}}{k}\right)^{1 / \alpha} \phi\left(\frac{k+2}{2^{q}}\right)\left[\frac{c_{2}}{1+\delta}\right]^{(1-\alpha) / \alpha}
$$

Lemma 1 now applies to show that

and hence

$$
P\left(A_{j k q}\right)=0(1) \frac{(q+2)^{1+\delta}}{2^{q\left(1+\frac{1}{2} \delta\right)}}
$$

$$
\sum_{q=1}^{\infty} \sum_{j=0}^{2 q} \sum_{k=[q / 3]}^{q} P\left(A_{j k q}\right)<\infty .
$$

The Borel-Cantelli lemma now shows that, for almost all $\omega$, there exists an integer $q(\omega)$ such that, whenever $q \geqq q(\omega), \omega \notin A_{j k q}$. That is $q \geqq q(\omega)$ implies

$$
T_{(j+k) / 2^{q}}(\omega)-T_{j / 2^{q}}(\omega)>\phi\left(\frac{k+2}{2^{q}}\right)\left[\frac{c_{2}}{1+\delta}\right]^{(1-\alpha) / \alpha}
$$

for all integers $k=[q / 3], \ldots, q$

$$
j=0, \ldots, 2^{q} .
$$

Now let $\eta(\omega)=q(\omega) / 2^{q(\omega)}$. For any $t$ satisfying $0 \leqq t \leqq 1$ and $h$ with $0<h<\eta(\omega)$ we define integers $q, j$ and $k$ as follows:

so that $q \geqq q(\omega)$,

$$
\frac{q+1}{2^{q+1}} \leqq h<\frac{q}{2^{q}},
$$

$$
\frac{j-1}{2^{q}}<t \leqq \frac{j}{2^{q}}<\frac{j+k}{2^{q}} \leqq t+h<\frac{j+k+1}{2^{q}},
$$

so that $[q / 3] \leqq k \leqq q$ and $0 \leqq j \leqq 2^{q}$. We now have

$$
\begin{aligned}
T_{t+h}(\omega)-T_{t}(\omega) & \geqq T_{(j+k) / 2^{q}}(\omega)-T_{j / 2^{q}}(\omega), & & \text { by the monotonicity of } T \\
& >\phi\left(\frac{k+2}{2^{q}}\right)\left[\frac{c_{2}}{1+\delta}\right]^{(1-\alpha) / \alpha}, & & \text { by } 3 \\
& >\phi(h)\left[\frac{c_{2}}{1+\delta}\right]^{(1-\alpha) / \alpha}, & & \text { by the monotonicity of } \phi .
\end{aligned}
$$

Thus

$$
\inf _{\substack{0 \leqq t \leqq 1 \\ 0<\bar{h}<\eta(\omega)}} \frac{T_{t+h}(\omega)-T_{t}(\omega)}{\phi(h)} \geqq\left[\frac{c_{2}}{1+\delta}\right]^{(1-\alpha) / \alpha}
$$

Since $P[\eta(\omega)>0]=1$, we have

$$
\lim _{\varepsilon \rightarrow 0} \inf _{\substack{0 \leqq t \leqq 1 \\ 0<h<\varepsilon}} \frac{T_{t+h}(\omega)-T_{t}(\omega)}{\phi(h)} \geqq\left[\frac{c_{2}}{1+\delta}\right]^{(1-\alpha) / \alpha}
$$


Now, letting $\delta$ tend to zero through a countable sequence, we obtain

almost surely.

$$
\lim _{\varepsilon \rightarrow 0} \inf _{\substack{0 \leqq t \leqq 1 \\ 0<h<\varepsilon}} \frac{T_{t+h}(\omega)-T_{t}(\omega)}{\phi(h)} \geqq c_{2}^{(1-\alpha) / \alpha}
$$

To obtain the opposite inequality we again take $\delta$ with $0<\delta<1$. Now let $A_{q j}$ be the event

and let

$$
T_{(j+1) / 2^{q}}(\omega)-T_{j / 2^{q}}(\omega) \leqq\left[\frac{c_{2}}{1-\delta}\right]^{(1-\alpha) / \alpha} \phi\left(\frac{1}{2^{q}}\right)
$$

$$
B_{q}=\bigcap_{j=0}^{2 q-1} A_{q j}^{c}
$$

Now, by the independent increment property,

which, by the stationarity of $T_{t}$,

$$
P\left(B_{q}\right)=\prod_{j=0}^{2 a-1} P\left(A_{q j}^{c}\right)
$$

$$
\begin{aligned}
& =\left[P\left(A_{q 0}^{c}\right)\right]^{2 q} \\
& =\left[1-P\left(A_{q 0}\right)\right]^{2 q} .
\end{aligned}
$$

Using $1-x \leqq \exp (-x)$ we obtain

$$
P\left(B_{q}\right) \leqq \exp \left[-2^{q} P\left(A_{q 0}\right)\right] .
$$

Lemma 1 shows that $2^{q} P\left(A_{q 0}\right) \rightarrow \infty$ as $q \rightarrow \infty$, so that $P\left(B_{q}\right) \rightarrow 0$ as $q \rightarrow \infty$. We thus have

as $q \rightarrow \infty$.

$$
P\left[\min _{0 \leqq j \leqq 2^{q}}\left(T_{(j+1) / 2^{q}}-T_{j / 2 q}\right) \leqq\left(\frac{c_{2}}{1-\delta}\right)^{(1-\alpha) / \alpha} \phi\left(\frac{1}{2^{q}}\right)\right] \rightarrow 1
$$

This, and the arbitrary choice of $\delta$, shows that

$$
\lim _{\varepsilon \rightarrow 0} \inf _{\substack{0 \leqq t \leq 1 \\ 0<h<\varepsilon}} \frac{T_{t+h}(\omega)-T_{t}(\omega)}{\phi(h)} \leqq c^{(1-\alpha) / \alpha}
$$

almost surely. (4) and (5) combine to prove the theorem.

Now let $X_{t}(\omega)$ be a stable process of index $\alpha, \alpha>1$, in $R$ and suppose that $\rho$ is given by 1 . We can now establish the following modulus of continuity result for the local time at zero of $X_{t}(\omega)$.

Theorem 2. Let $A_{u}(\omega)$ be the local time at zero of $X_{t}(\omega)$ and let $\psi(g)=$ $g^{1-1 / \alpha}(\log 1 / g)^{1 / \alpha}$. Then we have

$$
\lim _{\varepsilon \rightarrow 0} \sup _{\substack{0 \leqq u \leqq 1 \\ 0<g<\varepsilon}} \frac{A_{u+g}(\omega)-A_{u}(\omega)}{\psi(g)}=c_{3}
$$

almost surely. $c_{3}$ is given by $c_{3}=\rho^{\gamma}\left[\frac{c_{2}(\gamma)}{\gamma}\right]^{1-\gamma}$ and $\gamma=1-1 / \alpha$. 
Proof. Let

$$
\theta(a)=a^{\gamma}\left[\frac{\gamma}{c_{2}(\gamma)} \log \frac{1}{a}\right]^{1-\gamma}
$$

and

then, as $h \rightarrow 0^{+}$,

$$
\phi(h)=\frac{h^{1 / \gamma}}{(\log 1 / h)^{(1-\gamma) / \gamma}}
$$

$$
\theta\left[c_{2}(\gamma)^{(1-\gamma) / \gamma} \phi(h)\right] \sim h .
$$

Thus if $B_{u}(\omega)$ is defined by $B_{u}(\omega)=\inf \left[t: T_{t}(\omega)>u\right]$, where $T_{t}(\omega)$ is the stable subordinator of index $\gamma$, the proof of Theorem 1 ensures that

$$
\lim _{\varepsilon \rightarrow 0} \sup _{\substack{0 \leqq s \leqq 1 \rho \\ 0<h<\varepsilon}} \frac{B_{s+h}(\omega)-B_{s}(\omega)}{\theta(h)}=1
$$

almost surely. If $S_{t}(\omega)=\inf \left[u: A_{u}(\omega)>t\right]$ we have, by Stone's result, $S_{t}(\omega)=\rho T_{t}(\omega)$.

Thus $B_{u / \rho}(\omega)=A_{u}(\omega)$ and, from (6),

$$
\lim _{\varepsilon \rightarrow 0} \sup _{\substack{0 \leqq u \leqq 1 \\ 0<g<\varepsilon}} \frac{A_{u+g}(\omega)-A_{u}(\omega)}{\theta(g / \rho)}=1
$$

almost surely. Now, as $g \rightarrow 0^{+}$,

and the theorem follows.

$$
\begin{aligned}
\theta(g / \rho) & \sim \frac{1}{\rho^{\gamma}}\left[\frac{\gamma}{c_{2}(\gamma)}\right]^{1-\gamma} g^{\gamma}\left(\log \frac{1}{g}\right)^{1-\gamma} \\
& =\frac{\psi(g)}{c_{3}}
\end{aligned}
$$

We can now deduce the following result.

Corollary. Let $A_{u}(\omega)$ be the local time at zero of the Brownian motion process. Then

almost surely.

$$
\lim _{\varepsilon \rightarrow 0} \sup _{\substack{0 \leqq u \leqq 1 \\ 0<g<\varepsilon}} \frac{A_{u+g}(\omega)-A_{u}(\omega)}{(g \log 1 / g)^{\frac{1}{2}}}=1
$$

Proof. If $\alpha=2$ we have $\gamma=\frac{1}{2}$ and $c_{2}\left(\frac{1}{2}\right)=\frac{1}{4}$. For Brownian motion (1) implies that $\rho=2$. The corollary follows immediately.

\section{Collision Sets}

We recall the definition of Hausdorff dimension. For any subset $E$ of $R$ and each pair $\alpha, \varepsilon>0$ we define $\Lambda_{\varepsilon}^{\alpha}(E)$ as

$$
A_{\varepsilon}^{\alpha}(E)=\inf \sum_{i}\left(\operatorname{diam} S_{i}\right)^{\alpha}
$$

where the infimum is taken over all covers of $E$ by sets, $S_{i}$, of diameter less than $\varepsilon$. Now let $\Lambda^{\alpha}(E)=\lim _{\varepsilon \rightarrow 0} \Lambda_{\varepsilon}^{\alpha}(E)$. Then $A^{\alpha}(E)$ is a metric outer measure and so, at least, 
the Borel sets are measurable. For any Borel set $B$ there exists a number $b$, the (Hausdorff) dimension of $B$, such that

$$
b=\sup \left[\alpha>0: \Lambda^{\alpha}(B)=\infty\right]=\inf \left[\alpha>0: \Lambda^{\alpha}(B)=0\right] .
$$

(Here we take the supremum of the empty set to be zero.) We write $b=\operatorname{dim}(B)$.

We now show how Theorem 1 can be used to obtain Hausdorff dimension results.

Lemma 2. Let $T_{t}(\omega)$ be a stable subordinator of index $\alpha$ and for any Borel subset $B$ of $[0, \infty)$ we define $T(B, \omega)=\left(x: x=T_{t}(\omega)\right.$ for some $\left.t \in B\right)$. Then

$$
P(\operatorname{dim} T(B, \omega) \geqq \alpha \operatorname{dim} B \text { for all } B)=1 .
$$

Proof. It is sufficient to prove (7) in the case where $\operatorname{dim} B>0$. Suppose $\omega$ is such that (2) holds, and take $k$ and $\theta$ so that $0<1 / k<\alpha$ and $0<\theta<\operatorname{dim} B$. Choose any $\varepsilon>0$ and let $\delta=\varepsilon^{1 / k}$. Now cover $T(B, \omega)$ by intervals $S_{i}$ of length $a_{i}<\delta$ and let

$$
\begin{aligned}
b_{i} & =\inf \left[t: T_{t}(\omega) \in S_{i}\right], \\
c_{i} & =\sup \left[t: T_{t}(\omega) \in S_{i}\right] .
\end{aligned}
$$

Let $I_{i}$ be the interval centre $\frac{1}{2}\left(b_{i}+c_{i}\right)$ and length $2\left(c_{i}-b_{i}\right)$. Then the $I_{i}$ cover $B$, and, if $\varepsilon$ is sufficiently small, we have

Hence,

$$
a_{i}^{1 / k} \geqq\left|c_{i}-b_{i}\right| \quad \text { for all } i
$$

$$
2^{\theta} \sum a_{i}^{\theta / k} \geqq \Sigma\left[2\left(c_{i}-b_{i}\right)\right]^{\theta} .
$$

The sum on the left hand side can be made as close to $2^{\theta} A_{\delta}^{\theta / k} T(B, \omega)$ as we please but the sum on the right hand side is always greater than $A_{2 \varepsilon}^{\theta}(B)$. So

$$
2^{\theta} \Lambda_{\delta}^{\theta / k} T(B, \omega) \geqq \Lambda_{2 \varepsilon}^{\theta}(B) .
$$

As $\varepsilon \rightarrow 0, \delta \rightarrow 0$ and the right hand side tends to infinity so that $A^{\theta / k} T(B, \omega)=\infty$ and $\operatorname{dim} T(B, \omega) \geqq \theta / k$. Now, by the choice of $\theta$ and $k$ we have

$$
\operatorname{dim} T(B, \omega) \geqq \alpha \operatorname{dim} B .
$$

This is true for any $B$ and any $\omega$ satisfying (2) so the lemma follows.

In [4] Jain and Pruitt established the following result.

Proposition. Suppose that $1<\alpha \leqq \beta \leqq 2$ and that $X_{t}(\omega)$ is a stable process of index $\beta$ in $R$. Let $Y_{t}(\omega)$ be a stable process of index $\alpha$, defined on the same space as $X_{t}(\omega)$ and independent of $X_{t}(\omega)$. We define $C(\omega)$, the collision set of $X_{t}(\omega)$ and $Y_{t}(\omega), b y$

$$
C(\omega)=\left[x: x=X_{t}(\omega)=Y_{t}(\omega) \text { for some } t\right]
$$

We then have

$$
\operatorname{dim} C(\omega)=\alpha(1-1 / \beta) \quad \text { almost surely. }
$$

Their arguments apply directly to show the following, slightly weaker, result. If $\alpha<1$ and $Y_{t}(\omega)$ is not a subordinator then

$$
\alpha(1-1 / \beta)=\sup [\theta: P(\operatorname{dim} C>\theta)>0] .
$$


In what follows we establish the stronger result in the case where $Y_{t}(\omega)$ is a subordinator.

Theorem 3. Suppose that $0<\alpha<1<\beta \leqq 2$ and that $T_{t}(\omega)$ is a stable subordinator of index $\alpha$. Let $X_{t}(\omega)$ be a stable process of index $\beta$, defined on the same space as $T_{t}(\omega)$ and independent of $T_{t}(\omega)$. If $C(\omega)$ is the collision set of $T_{t}(\omega)$ and $X_{t}(\omega)$ then

$$
\operatorname{dim} C(\omega)=\alpha(1-1 / \beta)
$$

almost surely.

Proof. Let

$$
\begin{aligned}
S(\omega) & =\left[t: x=X_{t}(\omega)=T_{t}(\omega) \text { for some } x\right] \\
& =\left[t: X_{t}(\omega)-T_{t}(\omega)=0\right] \\
& =\left[t: Y_{t}(\omega)=0\right]
\end{aligned}
$$

where $Y_{t}(\omega)=X_{t}(\omega)-T_{t}(\omega)$.

It is easily shown that $Y_{t}(\omega)$ is a process with stationary independent increments such that

$$
\operatorname{Re} \log E \exp i\left(z Y_{t}\right) \sim-c t|z|^{\beta} \quad \text { as }|z| \rightarrow \infty .
$$

Under these circumstances Blumenthal and Getoor [2: p. 64] have shown that

$$
\operatorname{dim} S(\omega)=1-1 / \beta
$$

almost surely. Now $C(\omega)=T[S(\omega), \omega]$, so that by Lemma 2 we have

almost surely.

$$
\operatorname{dim} C(\omega) \geqq \alpha(1-1 / \beta)
$$

We will now indicate how the arguments of [4] can be modified to obtain the opposite inequality. For the remainder of the paper we shall assume the notions of polar sets contained in [4].

Let $f_{\beta}(t, x)$ and $f_{\alpha}(t, x)$ be the transition densities of $X_{t}$ and $T_{t}$ respectively. Then, see [7], there are positive constants $c_{4}, c_{5}$ and $c_{6}$ such that

$$
\begin{aligned}
c_{4} \leqq f_{\beta}(1, x) \leqq c_{5} & \text { if }|x| \leqq 1 \\
f_{\alpha}(1, x) \leqq c_{6} & \text { if }|x| \leqq 1
\end{aligned}
$$

whilst

$$
f_{\alpha}(1, x)=0 \quad \text { if and only if } x \leqq 0 .
$$

Let $Z_{t}(\omega)=\left[T_{t}(\omega), X_{t}(\omega)\right], z=(x, y)$ and

$$
U(z)=\int_{0}^{\infty} f_{\alpha}(t, x) f_{\beta}(t, y) d t .
$$

The following lemma plays the same role as Lemma 3.2 of [4].

Lemma 3. Define

$$
\begin{aligned}
k(x) & =x^{\frac{1}{1-\alpha(1-1 / \beta)}} & & \text { if } x>0 \\
& =0 & & \text { if } x \leqq 0 .
\end{aligned}
$$


Let $z$ be the point on the diagonal in $R^{2}$ whose polar coordinates are $\left(r, \frac{1}{4} \pi\right)$. Then there is a positive constant $c_{7}$ such that

$$
U(z) \geqq c_{7} k(r)
$$

whenever $|z|=|r|<\sqrt{2}$.

Proof. Suppose that $z$ has cartesian coordinates $(x, x)$. If $x \leqq 0,(9)$ is trivial since both sides of the inequality are zero. So we may suppose that $0<x<1$. Now, by the scaling property,

$$
U(z) \geqq \int_{x^{\alpha}}^{\infty} t^{-(1 / \alpha)-(1 / \beta)} f_{\alpha}\left(1, t^{-(1 / \alpha)} x\right) f_{\beta}\left(1, t^{-(1 / \beta)} x\right) d t .
$$

Since $0<x<1, t>x^{\alpha}$ implies that $t^{-(1 / \beta)} x<1$ so that

$$
U(z) \geqq c_{4} \int_{x^{\alpha}}^{\infty} t^{-(1 / \alpha)-(1 / \beta)} f_{\alpha}\left(1, t^{-(1 / \alpha)} x\right) d t .
$$

Putting $s=t^{-(1 / \alpha)} x$ we obtain

$$
U(z) \geqq x^{\frac{1}{1-\alpha(1-1 / \beta)}} \alpha c_{4} \int_{0}^{1} \frac{f_{\alpha}(1, s)}{s^{\alpha(1-1 / \beta)}} d s .
$$

Since $0<\alpha(1-1 / \beta)<1$ the lemma follows immediately.

We can now argue as in Theorem 3.1 of [4] to prove:

Lemma 4. Let $B$ be a Borel subset of the diagonal in $R^{2}$, and let $B$ be non polar for $Z_{t}(\omega)$. Then, if we consider $B$ as a subset of the line, $B$ has positive $k$-capacity.

Now let $Y_{t}\left(\omega^{\prime}\right)$ be a symmetric stable process of index $\theta$, defined on $\left(\Omega^{\prime}, \mathscr{F}{ }^{\prime}, P^{\prime}\right)$ and taking values in the diagonal. Define $\Gamma$ by

$$
\Gamma=\left[\left(\omega, \omega^{\prime}\right): Y_{t}\left(\omega^{\prime}\right)=Z_{s}(\omega) \text { for some } t, s>0\right] .
$$

Jain and Pruitt assert that $\Gamma \in \mathscr{F} \times \mathscr{F}^{\prime}$ but this is not clear. It seems better to consider the set $\Pi$ defined by

$$
\Pi=\left[\left(\omega, \omega^{\prime}\right): Y_{t-}\left(\omega^{\prime}\right) \text { or } Y_{t}\left(\omega^{\prime}\right)=Z_{s-}(\omega) \text { or } Z_{s}(\omega) \text { for some } s, t>0\right] .
$$

Then, if $S_{k q}=\left[(x, x): \frac{k-1}{2^{q}} \leqq x \leqq \frac{k+1}{2^{q}}\right]$ and $\Sigma_{k q}$ is the sphere in $R^{2}$ with $S_{k q}$ as diameter, we have

$$
\Pi=\bigcup_{n=1}^{\infty} \bigcup_{j=1}^{\infty} \bigcap_{q=1}^{\infty} \bigcup_{k=1}^{j^{2^{q}}} A_{k q n} \times B_{k q n}
$$

where

$$
A_{k q n}=\left[\omega: Z_{t-}(\omega) \text { or } Z_{t}(\omega) \in \Sigma_{k q} \text { for some } t>1 / n\right]
$$

and

$$
B_{k q n}=\left[\omega^{\prime}: Y_{t-}\left(\omega^{\prime}\right) \text { or } Y_{t}\left(\omega^{\prime}\right) \in S_{k q} \text { for some } t>1 / n\right]
$$


Then $A_{k q n} \in \mathscr{F}$ and $B_{k q n} \in \mathscr{F}^{\prime}$ (see [1:p. 59]) and hence

$$
\Pi \in \mathscr{F} \times \mathscr{F}^{\prime} .
$$

We now make three observations.

I. If $X_{t}(\omega)$ is one of the processes we are considering and $B$ is a Borel set we have

$$
\begin{aligned}
& P\left[\omega: X_{t}(\omega) \in B \text { for some } t>0\right] \\
& \quad=P\left[\omega: X_{t-}(\omega) \text { or } X_{t}(\omega) \in B \text { for some } t>0\right]
\end{aligned}
$$

(see [1: p. 59]).

II. $k(y-x)$ is proportional to the potential kernel of a stable subordinator of index $\alpha(1-1 / \beta)$ (see [1: p. 264]).

III. A set is polar for a stable subordinator of index $\gamma, \gamma<1$ if and only if it is polar for a symmetric stable process of index $\gamma$ (see [6]).

The proof of the theorem can now be completed by the arguments of Jain and Pruitt.

This result is interesting because the collisions take place at small times. For the collision set of recurrent processes Jain and Pruitt essentially showed that sooner or later the dimension of the collision set became arbitrarily close to $\alpha(1-1 / \beta)$. For the processes we have considered the collision set actually takes the dimension $\alpha(1-1 / \beta)$.

\title{
References
}

1. Blumenthal, R. M., Getoor, R. K.: Markov processes and potential theory. New York: Academic Press 1968.

2. - - Local times for Markov processes. Z. Wahrscheinlichkeitstheorie verw. Geb. 3, 50-74 (1964).

3. Boylan, E. S. : Local times for a class of Markov processes. Illinois J. Math. 8, 19-39 (1964).

4. Jain, N., Pruitt, W. E. : Collisions of stable processes. Illinois J. Math. 13, 241-248 (1969).

5. Lévy, P.: Théorie de l'Addition des Variables Aléatoires. Paris: Gauthier Villars 1937.

6. Orey, S.: Polar sets for processes with stationary independent increments. Proceedings of the Madison Conference on Markov Processes and Potential Theory. New York: John Wiley and Sons 1967.

7. Skohorod, A. V.: Asymptotic formulas for stable distribution laws. Select. Transl. math. Statist. Probab. 157-161 (1961).

8. Stone, C.: The set of zeros of a semi stable process. Illinois J. Math. 7, 631-637 (1963).

\author{
John Hawkes \\ University of Michigan \\ Mathematics Department \\ Ann Arbor, Michigan 48104, USA
}

